\title{
A Prospective Comparative Study of Different Methods of Cranioplasty: Our Institutional Experience
}

\author{
Hemant Sable ${ }^{1, \odot} \quad$ Mukesh P. Patel $^{1} \quad$ Kalpesh B. Shah ${ }^{1}$
}

Address for correspondence Hemant Sable, MCh, Samruddhi, Plot no. 113, Kotwal Nagar, Karjat 410201, District-Raigad, Maharashtra, India (e-mail: dr.hemants44@yahoo.com).

Indian J Neurosurg 2019;8:17-23

\begin{abstract}
Keywords

- autologous cranioplasty

- titanium mesh cranioplasty

- methods of cranioplasty

Introduction Cranioplasty is the surgical repair, reconstruction, and replacement of a removed part of the cranium, thus restoring its shape, symmetry, contour, and continuity, which is extremely important from a cosmetic as well as a psychosocial point of view. Continuing advances in cranioplasty techniques have enabled the repair of large and increasingly complicated calvarial defects; however, the optimal reconstructive material for different clinical scenarios still remains unclear and debatable.

Aim The aim of this study was to compare risk factors, complications, and the need for reoperation associated with different methods of cranioplasty, which are implemented in our institute.

Materials and Methods This study was a prospective study conducted between August 2016 and January 2019 in a tertiary institute. Sixty patients were studied and divided into three groups of which group 1 included 18 patients who underwent cranioplasty using ETO (ethylene oxidation)-sterilized autologous bone graft, group 2 included 17 patients who underwent cranioplasty using autologous bone graft placed in subcutaneous pocket, and group 3 included 25 patients who underwent titanium mesh cranioplasty. Data were collected and statistical analysis was performed.

Result and Conclusion Of the three groups studied, postoperative complications were more in group 1(ETO-sterilized autologous bone graft) and group 3 (titanium mesh cranioplasty) but the difference between the three groups was not statistically significant. Factors such as age, gender, initial diagnosis, interval between decompression craniectomy and cranioplasty, operative time, blood loss, method of fixation, and defect size had no statistically significant effect on postoperative outcomes.
\end{abstract}

\section{Introduction}

Cranioplasty is the surgical repair, reconstruction, and replacement of a removed part of the cranium, thus restoring its shape, symmetry, contour, and continuity, which is extremely important from a cosmetic as well as a psychosocial point of view. At the same time, it is also the cranial shield or barrier, thus affording protection to the underlying brain that would otherwise be vulnerable to injury. In this way, cranioplasty brings about a morphological as well as functional rehabilitation of the cranial vault. ${ }^{1}$ Large cranial defects also allow the atmospheric pressure to compress the unprotected intracranial contents, giving it a typical kidney bean appearance on CT, and more importantly adversely affecting brain perfusion, leading to sensory-motor deficits and a variety of symptoms such as headache, dizziness, seizures, anxiety attacks, depression, memory loss, and mood swings, all typical features of the "motor trephine" or "sinking skin flap" syndrome. ${ }^{2}$ Cranioplasty is a well-accepted neurosurgical procedure, which has applications in a wide range of postcraniectomy defects, resulting from cases of cerebral decompressive procedures following traumatic brain injury (TBI), ablative surgical resection of tumors, and so on. Continuing advances in
DOI https://doi.org/

10.1055/s-0039-3402929 ISSN 2277-954X.
License terms

(요 (1) $\Theta \circledast$ 
cranioplasty techniques have enabled the repair of large and increasingly complicated calvarial defects; however, the optimal reconstructive material for different clinical scenarios still remains unclear and debatable.

Given the varied need for both autologous and synthetic cranial graft materials in different clinical situations and scenarios, it is important to compare and establish benefits and shortfalls of both and compare rates of procedural complications and postoperative short and long-term outcomes between these two major cranioplasty techniques. Both alloplastic three-dimensional dynamic titanium mesh implants and autogenous calvarial bone grafts have been used extensively for cranial defect reconstruction. In the past, the choice of method has usually been subjective, based almost solely on the surgeon's preference and the cost factor involved. It has remained unclear, however, whether one method is superior to the other.

\section{Aims and Objectives}

This study aimed to compare the risk factors, complications, and need for reoperation associated with different methods of cranioplasty that are implemented in our institute.

\section{Materials and Methods}

\section{Place and Area of Study}

All patients posted for elective cranioplasty at our tertiary institute in the department of neurosurgery were studied. This was a prospective comparative observational study that was conducted from August 2016 to January 2019.

\section{Inclusion criteria were:}

1) Patients of both sex between 18 and 80 years of age.

2) Patients who required cranial defect reconstruction.

3) Cranioplasty reconstruction by autologous bone and titanium mesh.

4) Craniotomy defect size of $>25 \mathrm{~cm}^{2}$.

5) Decompression craniectomy performed for subdural hematoma (SDH), epidural hematoma (EDH), contusion, spontaneous intraparenchymal hematoma (IPH), depressed skull fractures, tumors, aneurysms, and arteriovenous malformations (AVMs).

6) Patients in whom multiple fragmented bone were found during initial craniectomy, bone fragments were discarded and the patient underwent titanium mesh cranioplasty later.

\section{Exclusion criteria:}

1) Patient affected by coagulopathy disorder.

2) Patient affected by uncontrolled diabetes/renal insufficiency.

3) Patient affected by autoimmune pathology.

4) Patient affected by immunosuppressive diseases/drugs.

5) Previously failed cranioplasty.

6) Craniectomy scar site infection.

\section{Methods}

Sixty patients were studied and divided into three groups of which group 1 included 18 patients who underwent cranioplasty using ETO (ethylene oxidation)-sterilized autologous bone graft, group 2 included 17 patients who underwent cranioplasty using autologous bone graft placed in subcutaneous pocket, and group 3 which included 25 patients who underwent titanium mesh cranioplasty.

\section{Cranioplasty Procedure}

Patients were administered Inj ceftriaxone $1 \mathrm{~g}$ preoperatively. Skin incision was taken at previous decompressive incision. Skin flap raised carefully to avoid breach of dura. Cranial bone edges were freed of soft tissue. When autoclaved titanium mesh was used, it was cut to the size of the defect, placed over the defect, and fixed to the surrounding bone with the help of mini plates and screw.

When autologous bone flap placed in the subcutaneous pocket was used (-Fig. 1A), a swab was taken from bone flap surface and sent for microbiological examination. Swab reports were obtained within 20 minutes. If swab report was negative for any organism, then the bone flap was used for cranioplasty. If the report was positive, then bone flap was discarded and titanium mesh cranioplasty ( - Fig. 1B) was performed. Once microbiological reports were negative, bone flap was thoroughly cleaned with saline, betadine solution, and hydrogen peroxide. Multiple holes were made over the autologous bone flap and on adjoining cranial bone, and fixation was performed using ethibond suture or stainless steel wire. At times, autologous bone was fixed to the surrounding bone with mini plates. Romo Vac suction drain no.12 was placed. Skin flap reposited back and incision sutured in two layers using vicryl 2.0 and ethilon 3.0. Sterile dressing was done.

In the postoperative period, IV Ceftriaxone was administered every 12 hours till postoperative day (POD) 5 , followed by oral antibiotics till POD 14. If the culture report of the initial negative swab comes positive, then antibiotics were started as per the sensitivity report. Suction drain was removed on POD 2 (provided drainage was in decreasing order). Patient with intraoperative blood loss $>300 \mathrm{~mL}$ were transfused with 1 unit of packed cell volume in immediate postoperative period. Suture removed on POD 14. Patients were followed-up at least 15 months and include clinical and CT examination. Appointments were scheduled at 6 weeks, 3 months, 6 months, 12 months, and 15 months.

Data collected included age, gender, initial diagnosis, interval between decompression craniectomy (DC) and cranioplasty, operative time, operative blood loss, defect size, and post-operative complications like surgical site infection (SSI), hematoma, seizures, sunken flap, removal of graft, bone resorption, and cosmetic outcome.

\section{Statistical Analysis}

Data were analyzed using Kruskal-Wallis test, MannWhitney test, and chi square test to calculate the $p$-value. A value $<0.05$ was considered statistically significant. 


\section{Results}

In group 1, 18 patients underwent ETO-sterilized autologous bone cranioplasty ( $n=18$ ), in group 2,17 patients underwent autologous bone cranioplasty using subcutaneously placed bone flap ( $n=17$ ), and in group 3, 25 patients underwent titanium mesh cranioplasty $(n=25)$.

Method of cranioplasty and postoperative outcome is given in - Table 1. Risk factors for cranioplasty and the effect of age on postoperative outcome are given in - Table $\mathbf{2}$. There was no statistical significance in postoperative outcomes in two different age groups. The effect of gender on postoperative outcome is given in - Table 3. There was no statistical significance in the postoperative outcome between males and females. The effect of initial diagnosis on postoperative outcome is given in - Table 4 . There was no effect of initial diagnosis on postoperative outcome. The effect of interval on postoperative outcome is given in - Table 5. There was no effect of interval on postoperative outcomes. The effect of operative time on postoperative outcome is given in - Table 6. There was no effect of operative time on postoperative outcome. The effect of blood loss on postoperative outcome is given in - Table 7 . There was no effect of intraoperative blood loss on postoperative outcome. The effect of defect size on postoperative outcome is given in - Table 8 . There was no effect of defect size on postoperative outcome. The effect of method of fixation on postoperative outcome is given in - Table 9. There was no effect of method of fixation on postoperative outcome.

\section{Discussion}

Cranioplasty (CP) is a commonly performed operation in the field of neurosurgery; however, it has a higher complication
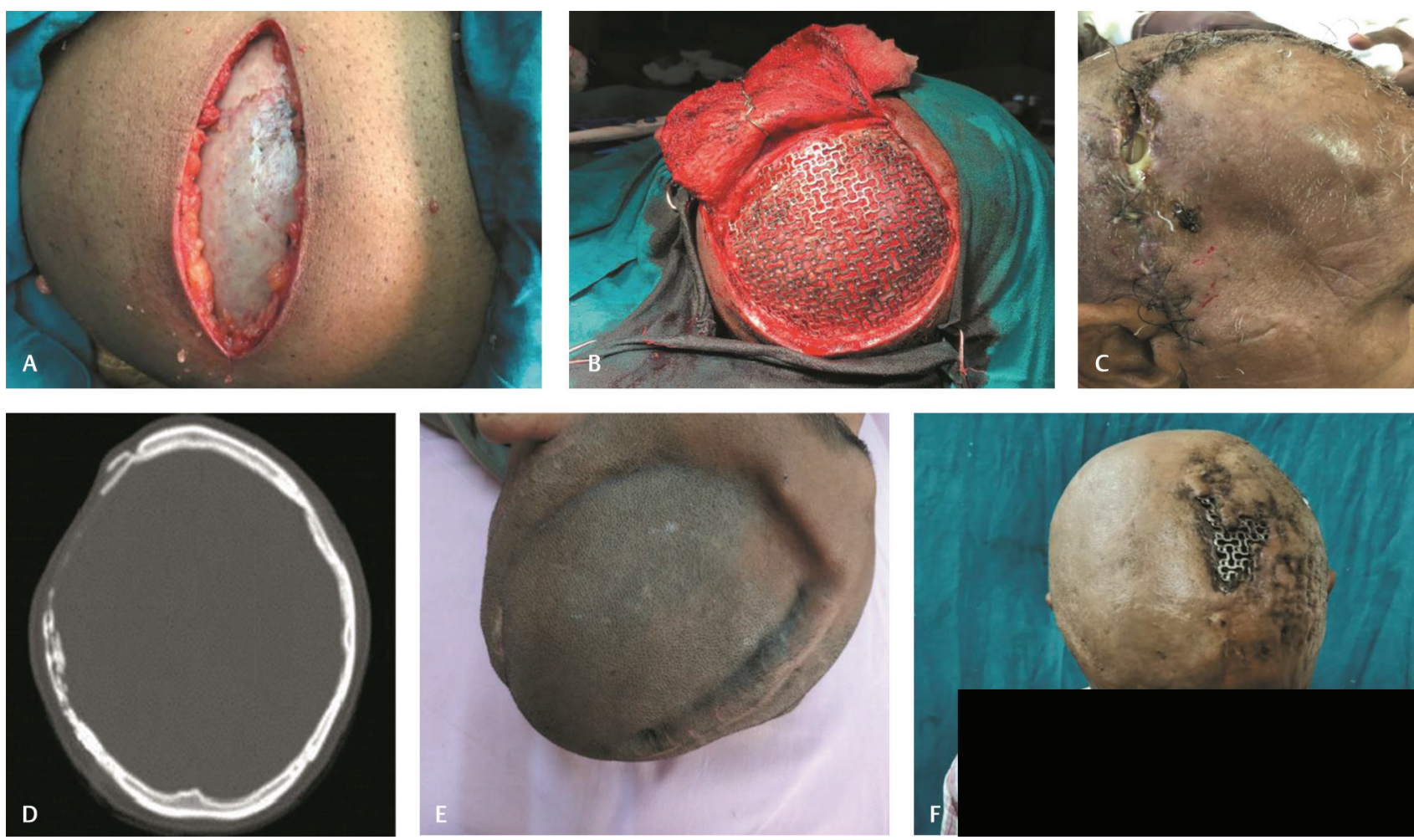

Fig. 1 (A) Autologous bone flap in subcutaneous pocket. (B) Titanium mesh cranioplasty. (C) Surgical site infection and wound dehiscence in patient with autologous bone Cranioplasty. (D) CT image of bone resorption. (E) Sunken bone flap. (F) Infected and exposed titanium mesh.

Table 1 Method of cranioplasty and postoperative outcome

\begin{tabular}{|l|l|l|l|l|}
\hline Post-operative outcome & $\begin{array}{l}\text { Group 1 } \\
\text { (autologous ETO) } \\
n=18\end{array}$ & $\begin{array}{l}\text { Group 2 } \\
\text { (autologous subcut) } \\
n=17\end{array}$ & $\begin{array}{l}\text { Group 3 } \\
\text { (titanium) } \\
n=25\end{array}$ \\
\hline Infection & 3 & 1 & 4 & \begin{tabular}{l}
$p$-Values \\
\hline Hematoma
\end{tabular} \\
\hline Seizures & 1 & 1 & 1 & 0.447 \\
\hline Sunken flap & 0 & 0 & 0 & 0.956 \\
\hline Bone resorption & 2 & 1 & 0 & 0.241 \\
\hline Removal & 3 & 2 & 5 & 0.099 \\
\hline Cosmesis (unsatisfactory) & 4 & 1 & 5 & 0.124 \\
\hline
\end{tabular}


20 Different Methods of Cranioplasty Sable et al.

Table 2 Effect of age on postoperative outcome

\begin{tabular}{|l|l|l|l|}
\hline Postoperative outcome & $\begin{array}{l}\text { Age (18-45 y) } \\
(\boldsymbol{n}=\mathbf{4 8})\end{array}$ & $\begin{array}{l}\text { Age (46-80 y) } \\
(\boldsymbol{n}=12)\end{array}$ & $p$-Value \\
\hline Infection & 7 & 2 & 0.631 \\
\hline Hematoma & 2 & 1 & 0.431 \\
\hline Seizures & 2 & 0 & 0.524 \\
\hline Sunken flap & 3 & 1 & 0.646 \\
\hline Bone resorption & 4 & 1 & 0.836 \\
\hline Removal & 8 & 2 & 0.539 \\
\hline Cosmesis (unsatisfactory) & 10 & 3 & 0.390 \\
\hline
\end{tabular}

Table 3 Effect of gender on postoperative outcome

\begin{tabular}{|l|l|l|l|}
\hline Postoperative outcome & Male $(\boldsymbol{n}=\mathbf{4 5})$ & Female $(\boldsymbol{n}=\mathbf{1 5})$ & $\boldsymbol{p}$-Value \\
\hline Infection & 5 & 4 & 0.147 \\
\hline Hematoma & 3 & 0 & 0.309 \\
\hline Seizures & 2 & 0 & 0.410 \\
\hline Sunken flap & 4 & 0 & 0.236 \\
\hline Bone resorption & 5 & 0 & 0.181 \\
\hline Removal & 7 & 3 & 0.692 \\
\hline Cosmesis (unsatisfactory) & 11 & 2 & 0.139 \\
\hline
\end{tabular}

Table 4 Effect of initial diagnosis on postoperative outcome

\begin{tabular}{|c|c|c|c|c|c|c|c|}
\hline $\begin{array}{l}\text { Postoperative } \\
\text { outcome }\end{array}$ & $\begin{array}{l}\text { IC hematoma } \\
(n=51)\end{array}$ & $\begin{array}{l}\text { Tumor } \\
(n=5)\end{array}$ & $\begin{array}{l}\text { MCA infarct } \\
(n=1)\end{array}$ & $\begin{array}{l}\text { Aneurysm } \\
(n=1)\end{array}$ & $\begin{array}{l}\text { AVM+ } \\
\text { IC hematoma } \\
(n=1)\end{array}$ & $\begin{array}{l}\text { Growing skull } \\
\text { no. }(n=1)\end{array}$ & $p$-Value \\
\hline Infection & 9 & 0 & 0 & 0 & 0 & 0 & 0.87 \\
\hline Hematoma & 2 & 0 & 0 & 0 & 0 & 1 & 0.702 \\
\hline Seizures & 1 & 1 & 0 & 0 & 0 & 0 & 0.458 \\
\hline Sunken flap & 3 & 1 & 0 & 0 & 0 & 0 & 0.884 \\
\hline Bone resorption & 4 & 1 & 0 & 0 & 0 & 0 & 0.940 \\
\hline Removal & 10 & 0 & 0 & 0 & 0 & 0 & 0.838 \\
\hline Cosmesis (unsatisfactory) & 12 & 1 & 0 & 0 & 0 & 0 & 0.958 \\
\hline
\end{tabular}

Abbreviations: AVM, arteriovenous malformation; IC, intercranial; MCA, middle cerebral artery.

Table 5 Effect of interval on postoperative outcome

\begin{tabular}{|l|l|l|l|}
\hline Postoperative outcome & $\begin{array}{l}\text { Interval ( } \mathbf{n} \text { months) } \\
\boldsymbol{n}=\mathbf{2 8}\end{array}$ & $\begin{array}{l}\text { Interval (>3 months) } \\
\boldsymbol{n}=32\end{array}$ & $\boldsymbol{p}$-Value \\
\hline Infection & 2 & 7 & 0.114 \\
\hline Hematoma & 3 & 0 & 0.060 \\
\hline Seizures & 0 & 2 & 0.182 \\
\hline Sunken flap & 2 & 2 & 0.891 \\
\hline Bone resorption & 2 & 3 & 0.757 \\
\hline Removal & 4 & 6 & 0.646 \\
\hline Cosmesis (unsatisfactory) & 6 & 7 & 0.797 \\
\hline
\end{tabular}


Table 6 Effect of operative time on postoperative outcome

\begin{tabular}{|c|c|c|c|}
\hline Postoperative outcome & $\begin{array}{l}\text { Operative time ( } \leq 120 \text { mins) } \\
n=55\end{array}$ & $\begin{array}{l}\text { Operative time (> } 120 \text { mins) } \\
n=5\end{array}$ & $p$-Value \\
\hline Infection & 8 & 1 & 0.856 \\
\hline Hematoma & 2 & 1 & 0.567 \\
\hline Seizures & 2 & 0 & 0.897 \\
\hline Sunken flap & 4 & 0 & 0.795 \\
\hline Bone resorption & 5 & 0 & 0.756 \\
\hline Removal & 9 & 1 & 0.897 \\
\hline Cosmesis (unsatisfactory) & 12 & 1 & 1.00 \\
\hline
\end{tabular}

Table 7 Effect of blood loss on postoperative outcome

\begin{tabular}{|l|l|l|l|}
\hline Postoperative outcome & $\begin{array}{l}\text { Blood loss }(\leq 300 \mathrm{~mL}) \\
n=52\end{array}$ & $\begin{array}{l}\text { Blood loss }(>300 \mathrm{~mL}) \\
n=8\end{array}$ & $p$-Value \\
\hline Infection & 7 & 2 & 0.742 \\
\hline Hematoma & 2 & 1 & 0.875 \\
\hline Seizures & 2 & 0 & 0.920 \\
\hline Sunken flap & 3 & 1 & 0.538 \\
\hline Bone resorption & 4 & 1 & 0.577 \\
\hline Removal & 8 & 2 & 0.786 \\
\hline Cosmesis (unsatisfactory) & 10 & 3 & 0.306 \\
\hline
\end{tabular}

Table 8 Effect of defect size on postoperative outcome

\begin{tabular}{|l|l|l|l|l|}
\hline Postoperative outcome & $\begin{array}{l}\text { Defect size } \begin{array}{l}\text { D } \\
n=6\end{array} \\
\text { Infection }\end{array}$ & $\begin{array}{l}\text { Defect size 75-125 } \mathrm{cm}^{2} \\
n=23\end{array}$ & $\begin{array}{l}\text { Defect size }>125 \mathrm{~cm}^{2} \\
n=31\end{array}$ & $\boldsymbol{p}$-Value \\
\hline Hematoma & 4 & 5 & 0.539 \\
\hline Seizures & 1 & 1 & 1 & 0.189 \\
\hline Sunken flap & 1 & 0 & 1 & 0.189 \\
\hline Bone resorption & 0 & 2 & 2 & 0.819 \\
\hline Removal & 0 & 2 & 3 & 0.699 \\
\hline Cosmesis (unsatisfactory) & 0 & 5 & 5 & 0.539 \\
\hline
\end{tabular}

Table 9 Effect of method of fixation on postoperative outcome

\begin{tabular}{|l|l|l|l|l|}
\hline Postoperative outcome & $\begin{array}{l}\text { SS Wire } \\
(\boldsymbol{n}=\mathbf{4})\end{array}$ & $\begin{array}{l}\text { Miniplates } \\
(\boldsymbol{n}=\mathbf{3 1})\end{array}$ & $\begin{array}{l}\text { Ethibond } \\
(\boldsymbol{n}=\mathbf{2 5})\end{array}$ & $\mathbf{2}$ \\
\hline Infection & 0 & 7 & 0 & 0.222 \\
\hline Hematoma & 1 & 2 & 0 & 0.093 \\
\hline Seizures & 0 & 2 & 3 & 0.386 \\
\hline Sunken flap & 1 & 0 & 4 & 0.066 \\
\hline Bone resorption & 1 & 0 & 0.060 \\
\hline Removal & 1 & 8 & 1 & 0.088 \\
\hline Cosmesis (unsatisfactory) & 2 & 7 & 4 & 0.291 \\
\hline
\end{tabular}

rate than other types of elective craniotomy. ${ }^{3}$ According to recent studies, the rates of complications after cranioplasty ranged from 16.4 to $34 \%$, and varied according to the type of procedure and materials used. ${ }^{4-6}$ Two of the most commonly used materials are autologous bone flaps and titanium mesh.
Although many clinicians have investigated what methods of autologous bone flap sterilization and preservation is better over several decades, there is currently no standard guideline for the sterilization and preservation of skull bone flaps for $\mathrm{CP}$. The most commonly used techniques for preserving 
the autologous bone flaps involve cryopreservation and subcutaneous implantation into the abdominal pocket. ${ }^{3}$ Most surgeons prefer subcutaneous pocket because they are of the opinion that keeping bone in the subcutaneous pocket will ensure viability of the bone which will result in better fusion and less infection rate.

In our institution, we place bone flap in the abdominal subcutaneous pocket or perform ethylene oxide sterilization. In our study, we looked for postoperative outcomes in each of the three groups.

In our study, the overall infection rate was 13.33\% (-Table 1), of which the maximum infection rate was seen in the group where ETO-sterilized autologous bone flap ( - Fig. 1C) was used (16.66\%), followed by group where titanium mesh cranioplasty (16\%) was performed. The least infection rate was seen in the group where subcutaneously placed autologous bone flap was used (5.8\%); however, the difference between the three groups was not statistically significant.

In the study conducted by Kim et $\mathrm{al}^{7}{ }^{7}$ the researchers compared complications following different methods of cranioplasty in 97 patients who underwent cranioplasty. The overall infection rate was $7.9 \%$ and the infection rate of the group that underwent sterilized autologous bone cranioplasty was $6.7 \%$.

In the study byMahapatra et al, ${ }^{8}$ wound infection was seen in $7.8 \%$ of patients who underwent autologous bone cranioplasty and $16.67 \%$ of patients who underwent synthetic material cranioplasty.

In our study, one case of postoperative hematoma was seen in each of the three groups (-Table 1). Group with titanium mesh cranioplasty and autologous bone cranioplasty using subcutaneous bone flap required removal of flap, whereas hematoma was managed conservatively in the ETO-sterilized group. Differences in the three groups with respect to hematoma were not statistically significant. In a study conducted by Kim et al, intracranial hematoma was observed in 3\% ( 1 in 30) of patients who had undergone autologous bone cranioplasty. In the study conducted by Mahapatra et al, where the group studied 129 patients who underwent cranioplasty following decompressive craniectomy in traumatic brain injury, intracranial hemorrhage was seen in $4.7 \%$ of patients who underwent autologous bone cranioplasty using subcutaneously placed bone flap and in $1.1 \%$ of patients who underwent cryopreserved autologous bone flap cranioplasty.

In our study, two cases of postoperative seizure were observed in the group with titanium mesh cranioplasty (8\%), which were managed conservatively ( - Table $\mathbf{1}$ ). No cases of seizure were seen in the other two groups. Differences in the three groups with respect to seizures were not statistically significant. In the study conducted by Mahapatra et al, seizures were observed in $2 \%$ of patients who underwent autologous bone cranioplasty and none in synthetic material cranioplasty. Similarly, in the study by Kim et al, seizures were seen in $1 \%$ of patients who underwent autologous bone cranioplasty

In our study, three cases of bone flap resorption were seen in ETO-sterilized group (16.66\%) ( - Fig. 1D) and two cases in the subcutaneous bone flap group (11.76\%), and the difference was statistically significant. In the study by Kim et al, bone resorption was observed in $60 \%$ of patients who underwent cryopreserved autologous bone cranioplasty. Similarly, in a study conducted by Jaakko et $\mathrm{al}^{9}$ who studied 100 patients of cranioplasty, bone resorption was seen in $15 \%$ of patients who underwent autologous bone cranioplasty.

In our study, two patients in the ETO-sterilized (11.1\%) group developed sunken flap ( - Fig. 1E), of which one required removal of the bone flap. One patient in the group with cranioplasty using subcutaneously placed bone flap (5\%) developed sunken flap which was managed conservatively (-Table 1). Differences among the three groups were not statistically significant. Sunken bone flap was observed in $2 \%$ of patients undergoing autologous cranioplasty in a study conducted by Mahapatra et al.

In our study, removal of cranioplasty material was seen in five patients of the group with titanium mesh cranioplasty (20\%) and in four patients of the ETO-sterilized group $(22.22 \%)$ ( - Table 1). A major reason for removal was infec-

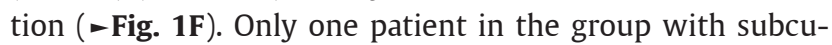
taneous-placed autologous bone cranioplasty (5\%) required removal of bone flap. Differences among the three groups were not statistically different. In the study by Mahapatra et al, removal of cranioplasty material was observed in $13.7 \%$ of patients who underwent autologous bone cranioplasty and in $16.66 \%$ of patients who underwent synthetic material cranioplasty. Most common reasons for removal in this study were wound infection and dehiscence. On the other hand, in the study by Jaakko et al, removal was seen in $30 \%$ of patients who underwent autologous bone cranioplasty and in $17 \%$ of patients who underwent synthetic material cranioplasty.

In our study, cosmetically unsatisfactory outcome was seen in five patients of titanium mesh cranioplasty (20\%), six patients with ETO-sterilized autologous bone cranioplasty (33.3\%), and in only two patients with subcutaneous autologous bone cranioplasty (11.76\%). The difference among the three groups was not statistically significant. Cosmetically unsatisfactory outcome was observed in 3.5\% of patients with synthetic material cranioplasty and $15 \%$ of patients with autologous cranioplasty in the study conducted by Jaakko et al. In the study conducted by Jeyaraj et al, ${ }^{10}$ cosmetically unsatisfactory outcome was seen in $5 \%$ of patients who underwent titanium mesh cranioplasty and in $50 \%$ of patients who had underwent autologous split thickness calvarial bone graft cranioplasty.

In our study, the average operative time across all three groups was 120 minutes and the blood loss was around $300 \mathrm{~mL}$ (-Tables 6 and 7). So, there was no technical difficulty in performing operative procedure among all three groups. In titanium mesh cranioplasty, we used mini plates as the method of fixation to the surrounding bone.

One of the aims of our study was to identify risk factors associated with the outcomes of cranioplasty. Schwartz et al ${ }^{11}$ and Martin et $\mathrm{al}^{12}$ found that being younger than 30 years was the risk factor for bone flap resorption; however, no such association was found in our study. Grant et $\mathrm{al}^{13}$ found higher bone resorption rates in bone flap larger than $75 \mathrm{~cm}^{2}$, but no 
such association was found in our study. Mracek et $\mathrm{al}^{3}$ found that fixation of bone flap by sutures was associated with higher rates of bone flap resorption as compared with mini plates; however, no such association was found in our study.

Sundseth et a ${ }^{14}$ found no statistically significant relationship between the time interval for decompressive craniectomy to cranioplasty and the risk of surgical site infection which is similar to our study (-Table 5). Complications were more in the group with interval $>3$ months, although the difference between the two groups was not statistically significant. Our findings were similar to that seen in the study conducted by Mahapatra et al.

Tokoro et $\mathrm{al}^{7}$ and Marcek et $\mathrm{al}^{3}$ found that longer operative time was a significant risk factor for surgical site infection, but no such association was seen in our study (-Table 6), which is similar to the findings of the study published by Kim et $\mathrm{al}^{15}$ and Lee et al. ${ }^{16}$ In the study by Kim et al, bone flap resorption occurred significantly more in patients who underwent decompressive craniectomy due to traumatic brain injury (8.5\%) than due to any other primary diagnosis, while in our study, bone flap resorption was seen in $7.8 \%$ of patients when the initial diagnosis was intracranial hematoma due to TBI ( - Table 4).

Thus, from our study, it is evident that factors such as age, gender, initial diagnosis, and the interval between decompressive craniectomy and cranioplasty, operative time, blood loss, defect size, and method of fixation, has no effect on outcomes of cranioplasty.

\section{Conclusion}

- Cranioplasty is performed not only for preserving normal appearance and physical barrier but also achieving neurologic outcome.

- This study brings out the fact that all the three methods are viable options for reconstruction of cranial defect.

- Of the three groups studied, postoperative complications were more in group 1(ETO-sterilized autologous bone graft) and group 3 (titanium mesh cranioplasty) but the difference among the three groups was not statistically significant.

- We conclude that considering low-complication rates, low cost, and better mechanical, biologic, and immunologic properties, autologous bone cranioplasty using subcutaneous placed bone flap would be the preferred option.

- In this study, age, gender, initial diagnosis, interval between decompression craniectomy and cranioplasty, operative time, blood loss, method of fixation, and defect size have no statistically significant effect on postoperative outcomes.

\section{Conflict of Interest}

None declared.

\section{References}

1 Aciduman A, Belen D. The earliest document regarding the history of cranioplasty from the Ottoman era. Surg Neurol 2007;68(3):349-352

2 Wallace RD, Salt C, Konofaos P. Comparison of Autogenous and Alloplastic Cranioplasty Materials Following Impact Testing. J Craniofac Surg 2015;26(5):1551-1557

3 Mracek J, Hommerova J, Mork J, Richtr P, Priban V. Complications of cranioplasty using a bone flap sterilised by autoclaving following decompressive craniectomy. Acta Neurochir (Wien) 2015;157(3):501-506

4 Bobinski L, Koskinen LO, Lindvall P. Complications following cranioplasty using autologous bone or polymethylmethacrylate-retrospective experience from a single center. Clin Neurol Neurosurg 2013;115(9):1788-1791

5 Chang V, Hartzfeld P, Langlois M, Mahmood A, Seyfried D. Outcomes of cranial repair after craniectomy. J Neurosurg 2010;112(5):1120-1124

6 Gooch MR, Gin GE, Kenning TJ, German JW. Complications of cranioplasty following decompressive craniectomy: analysis of 62 cases. Neurosurg Focus 2009;26(6):E9

7 Kim SH, Kang DS, Cheong JH, Kim JH, Song KY, Kong MH. Comparison of complications following cranioplasty using a sterilized autologous bone flap or polymethyl methacrylate. Korean J Neurotrauma 2017;13(1):15-23

8 Basheer N, Gupta D, Mahapatra A, Gurjar H. Cranioplasty following decompressive craniectomy in traumatic brain injury : Experience at Level - I apex trauma centre. Indian J Neurotrauma. 2010;7(2):139-144

9 Piitulainen JM, Kauko T, Aitasalo KM, Vuorinen V, Vallittu PK, Posti JP. Outcomes of cranioplasty with synthetic materials and autologous bone grafts. World Neurosurg; 2015 83(5)83(5) 708-714708-714

10 Jeyaraj CP. Reconstruction of large calvarial defects using titanium mesh versus autologous split thickness calvarial bone grafts: a comprehensive comparative evaluation of the two major cranioplasty techniques. J Maxillofac Oral Surg 2018;17(3):308-323

11 Schwarz F, Dünisch P, Walter J, Sakr Y, Kalff R, Ewald C. Cranioplasty after decompressive craniectomy: is there a rationale for an initial artificial bone-substitute implant? A single-center experience after 631 procedures. J Neurosurg 2015;124(3):710-7155

12 Martin KD, Franz B, Kirsch M, et al. Autologous bone flap cranioplasty following decompressive craniectomy is combined with a high complication rate in pediatric traumatic brain injury patients. Acta Neurochir (Wien) 2014;156(4):813-824

13 Grant GA, Jolley M, Ellenbogen RG, Roberts TS, Gruss JR, Loeser JD. Failure of autologous bone-assisted cranioplasty following decompressive craniectomy in children and adolescents. J Neurosurg 2004; 100(2, Suppl Pediatrics)163-168

14 Sundseth J, Sundseth A, Berg-Johnsen J, Sorteberg W, Lindegaard KF. Cranioplasty with autologous cryopreserved bone after decompressive craniectomy: complications and risk factors for developing surgical site infection. Acta Neurochir (Wien) 2014;156(4):805-811, discussion 811

15 Kim JS, Cheong JH, Ryu JI, Kim JM, Kim CH. Bone flap resorption following cranioplasty after decompressive craniectomy: preliminary report. Korean J Neurotrauma 2015;11(1):1-5

16 Lee CH, Chung YS, Lee SH, Yang HJ, Son YJ, Korea S. Analysis of the factors influencing bone graft infection after cranioplasty. J Trauma Acute Care Surg 2012;73(1):255-260 